\title{
The comparison of endothelial function between conduit artery and microvasculature in patients with coronary artery disease
}

\author{
Emi Tajima, Masashi Sakuma, Seiko Tokoi, Hisae Matsumoto, Fumiya Saito, \\ Ryo Watanabe, Shigeru Toyoda, Shichiro Abe, Teruo Inoue
}

Department of Cardiovascular Medicine, Dokkyo Medical University School of Medicine, Mibu, Japan

\begin{abstract}
Background: Flow-mediated dilation (FMD) and reactive hyperemia-peripheral arterial tonometry (RH-PAT) are both established modalities to assess vascular endothelial function. However, clinical significance of FMD and RH-PAT may be different because these methods measure vascular function in different vessels (conduit arteries and resistance vessels).

Methods: To elucidate differences in the clinical significance of FMD and RH-PAT, a simultaneous determination of FMD was performed and reactive hyperemia index (RHI) measured by RH-PAT in 131 consecutive patients who underwent coronary angiography for suspicion of coronary artery disease (CAD). Results: There was no significant correlation between FMD and RHI in patients overall. When patients were divided into four groups: $F M D \geq 6 \% / R H I \geq 1.67$ group, $F M D \geq 6 \% / R H I<1.67$ group, $F M D<6 \% / R H I \geq 1.67$ group and $F M D<6 \% / R H I<1.67$ group, the highest incidence of multivessel CAD was seen in the FMD $<6 \% / R H I<1.67$ group (52\%). Multiple logistic regression analysis showed that a prevalence of both $F M D<6 \%$ and RHI $<1.67$ was an independent predictor of multivessel CAD (odds ratio: $4.160,95 \%$ confidence interval: $1.505-11.500, p=0.006$ ). RHI was negatively correlated with the baseline vessel diameter $(R=-0.268, p=0.0065)$ and maximum vessel diameter $(R=-0.266, p=0.0069)$ in patients with $F M D<6 \%$, whereas these correlations were absent in patients with $F M D \geq 6 \%$.

Conclusions: Present results suggest that noninvasive assessment of vascular endothelial functions provide pathophysiological information on both conduit arteries and resistance vessels in patients with CAD. (Cardiol J 2020; 27, 1: 38-46)
\end{abstract}

Key words: flow mediated-dilation, reactive hyperemia-peripheral arterial tonometry, reactive hyperemia index, vascular endothelial function, coronary artery disease

\section{Introduction}

The endothelium plays a seminal role in the regulation of vascular tone, new vessel growth, thrombogenicity, and inflammation [1]. Moreover, endothelial dysfunction is associated with cardiovascular events. Brachial artery flow-mediated dilation (FMD) and reactive hyperemia-peripheral arterial tonometry (RH-PAT) are established methods to assess vascular endothelial function. FMD measures the ability of the brachial artery to re- lease endothelial nitric oxide (NO) during reactive hyperemia after a 5-min occlusion of the artery with a blood pressure cuff $[2,3]$. Celermajer et al. [4] first observed this response in vivo by using ultrasound to measure changes in the diameter of the brachial artery, and later demonstrated this response to be mainly NO-dependent [5]. Importantly, peripheral vascular endothelial function as assessed by FMD correlates with coronary artery endothelial function [6]. In addition, FMD has independent prognostic value to predict future

Address for correspondence: Masashi Sakuma, MD, PhD, Department of Cardiovascular Medicine, Dokkyo Medical University School of Medicine, 880 Kitakobayashi, Mibu, Tochigi, Japan 321-0293, tel: 81-282-86-1111, fax: 81-282-86-5633, e-mail: masakuma@dokkyomed.ac.jp

Received: 8.03.2018 Accepted: 21.06.2018 
cardiovascular events that may exceed the predictive ability of traditional risk factors [7]. On the other hand, RH-PAT has been recently introduced as a new surrogate marker to evaluate endothelial function. RH-PAT is a noninvasive, automatic, and quantitative method of clinical assessment based on digital measurements of the hyperemic response [8]. Although FMD reflects endothelial function of large conduit arteries [9]. Reactive hyperemia index (RHI) as measured by RH-PAT reflects endothelial function of the microvasculature (i.e., resistance vessels) [10] and depends more on endothelium-derived hyperpolarizing factor (EDHF) than NO [11, 12]. However, endothelial function measured by RH-PAT also predicts cardiovascular events [13].

Although both FMD and RH-PAT can predict cardiovascular events, it was hypothesized that the clinical significance of these two vascular endothelial function tests in patients with cardiovascular diseases are different because these methods measure vascular function in different vessels (conduit arteries or resistance vessels). In this study, both FMD and RH-PAT-based RHI were simultaneously measured in patients with coronary artery disease (CAD) to elucidate differences in clinical significance of each method for assessment of vascular endothelial function.

\section{Methods}

\section{Subjects and study outline}

Simultaneous measurement of FMD and RH-PAT were performed in 131 consecutive patients, who underwent diagnostic coronary angiography due to suspicion of CAD (including stable angina pectoris, old myocardium infarction, coronary spastic angina and chest pain syndrome) at Dokkyo Medical University Hospital. Patients were excluded if they had acute coronary syndrome, valvular heart disease, atrial fibrillation/flutter, permanent pacemaker implantation, impaired left ventricular systolic function (left ventricular ejection fraction $<40 \%$ ), aortic dissection, malignancy or serious liver diseases, or were on hemodialysis. The Dokkyo Medical University review board approved the study protocol, and written informed consent was obtained from each patient.

\section{Simultaneous measurement of FMD and RHI}

Simultaneous measurement of FMD and RHI was performed in the morning of the day before coronary angiography, according to the method previously described by Tomiyama et al. [14]. In brief, the subjects were instructed to fast overnight and to abstain from alcohol, smoking, caffeine and antioxidant vitamins for at least $12 \mathrm{~h}$ before the measurements. They were asked to rest in the sitting position in a quiet, dark, air-conditioned room $\left(22^{\circ} \mathrm{C}\right.$ to $\left.25^{\circ} \mathrm{C}\right)$ for $5 \mathrm{~min}$. Then, they were asked again to rest for at least $15 \mathrm{~min}$ in the supine position in the same room before FMD and RH-PAT procedures. Blood pressure was measured in the left arm using a mercury sphygmomanometer with an appropriately sized cuff and recorded to the nearest $2 \mathrm{mmHg}$. After blood pressure was measured, a $10-\mathrm{MHz}$ linear array ultrasound transducer (Unex EF 18G, UNEX Corp., Nagoya, Japan) was placed on the proximal right brachial artery to measure FMD, and the manchette was rolled at the forearm. For the RH-PAT procedure (EndoPAT-2000, Itamar Medical Ltd., Caesarea, Israel), a peripheral arterial tonometry probe was placed on the right index finger and a control tonometry probe was also placed on the left index finger to eliminate sympathetic nerve effects. The RH-PAT probes were exchanged for each patient. For FMD measurement, ultrasound longitudinal images were recorded at baseline and continuously from $30 \mathrm{~s}$ before to $\geq 2$ min after cuff deflation following compression with a cuff pressure that was $50 \mathrm{mmHg}$ above the systolic blood pressure of the right forearm for $5 \mathrm{~min}$. The diastolic diameter of the brachial artery was determined semi-automatically using an instrument equipped with software for monitoring the brachial artery diameter. FMD was estimated as percent change of brachial artery diameter at maximal dilation during hyperemia compared with the baseline value. In the RH-PAT procedure, the RHI value was calculated as the ratio of reactive hyperemia between two hands. Moreover, other parameters obtained during the FMD procedure such as baseline vessel diameter, maximum vessel diameter and blood flow velocity increase (maximum blood flow velocity/baseline blood flow velocity) were also measured after forearm cuff deflation following compression.

\section{Assessment of the results of coronary angiography}

The angiographic findings were visually assessed for all atherosclerotic coronary lesions by an investigator who was unaware of the study design. According to the classification of the American Heart Association, the percent diameter stenosis was evaluated for each lesion and the lesion location was assessed. The number of affected vessels 
was assessed, considering that $\geq 75 \%$ diameter stenosis was a significant atherosclerotic coronary lesion. If there were no significant stenotic lesions, provocation of coronary artery spasm was performed with an intracoronary injection of acetylcholine. Acetylcholine chloride was injected in incremental doses of 25 and $50 \mu \mathrm{g}$ into the right coronary artery and of 25,50 , and $100 \mu \mathrm{g}$ into the left coronary artery over $20 \mathrm{~s}$, with at least a $3 \mathrm{~min}$ interval between each injection. The patients who had a positive acetylcholine test were diagnosed with coronary spastic angina and those with a negative acetylcholine test were diagnosed with chest pain syndrome.

\section{Coronary risk factor assessment}

Prior to FMD and RH-PAT procedures, information on coronary risk factors such as hypertension, diabetes, dyslipidemia and smoking habit were obtained from each patient, as well as information on medication usage. Height and body weight were measured, and body mass index (BMI) was calculated as body weight $(\mathrm{kg}) /(\text { height }[\mathrm{m}])^{2}$. Blood pressure was measured using a mercury sphygmomanometer with an appropriately sized cuff and recorded to the nearest $2 \mathrm{mmHg}$. Just after FMD and RH-PAT procedures, peripheral blood samples were taken via the antecubital vein. Serum creatinine level was measured using an enzymatic method, and the estimated glomerular filtration rate (eGFR) was calculated by a formula provided by the Japanese Society of Nephrology Chronic Kidney Disease (CKD) Practice Guide: eGFR $\left(\mathrm{mL} / \mathrm{min} / 1.73 \mathrm{~m}^{2}\right)=194 \times($ serum creatinine level $[\mathrm{mg} / \mathrm{dL}])^{-1.094} \times(\text { age }[\mathrm{y}])^{-0.287}$. The product of this equation was multiplied by a correction factor of 0.739 for women [15]. Total cholesterol (TC) and triglyceride (TG) levels were determined using enzymatic methods, high-density lipoprotein cholesterol (HDL-C) was measured using the precipitation method and low-density lipoprotein cholesterol (LDL-C) was calculated using the Friedewald formula: LDL-C = TC - HDL-C - (TG/5). The LDL-C could not be calculated in those patients with a triglyceride level over $400 \mathrm{mg} / \mathrm{dL}$. Hemoglobin A1c was measured by high-performance liquid chromatography and values were expressed according to the National Glycohemoglobin Standardization Program.

\section{Statistical analysis}

Data were expressed as the mean \pm standard deviation (SD) or median and interquartile range. Normality for distribution of continuous variables was assessed using the Shapiro-Wilk test. Multiple group comparisons were performed using one-way analysis of variance followed by the post-hoc Bonferroni test for continuous variables and the Fisher exact test for categorical variables. Intra-group comparisons for normally distributed continuous variables were performed using an unpaired $t$ test. The correlation between two variables was determined by the Pearson correlation coefficient. Logistic regression analysis was performed to predict multi-vessel CAD using age, gender, various risk factors and vascular endothelial function parameters as independent variables. Variables that could predict multi-vessel disease using a simple logistic regression model were first we determined, then multiple regression analysis was performed using the variables identified by the simple logistic regression model. All statistical analyses were performed using the statistical package for Social Science (Dr. SPSS II for Windows, SPSS Inc., Tokyo, Japan). $\mathrm{P}<0.05$ was considered significant.

\section{Results}

\section{Relationship between both values of FMD and RHI}

In all 131 patients, there was no significant correlation between FMD and RHI $(\mathrm{R}=0.119)$. Then, the patients were divided into four groups, based on cut off values of $6 \%$ for FMD and 1.67 for RHI, according to previous reports $[16,17]$, i.e., FMD $\geq 6 \% /$ RHI $\geq 1.67$ group $(\mathrm{n}=22 ; 17 \%)$, FMD $\geq 6 \% / \mathrm{RHI}<1.67$ group $(\mathrm{n}=7 ; 5 \%), \mathrm{FMD}<6 \% /$ $/ \mathrm{RHI} \geq 1.67$ group $(\mathrm{n}=81 ; 62 \%)$ and $\mathrm{FMD}<6 \% /$ $/$ RHI $<1.67$ group $(\mathrm{n}=21 ; 6 \%)$ (Fig. 1$)$. Baseline characteristics were compared among these four groups. There were no significant differences among the four groups in age, sex, traditional risk factors and medications. All patients in groups with RHI $<1.67$ had significant coronary artery stenosis. In the FMD <6\%/RHI $\geq 1.67$ group, $86 \%$ of patients had stable angina. The incidence of old myocardial infarction and multi-vessel CAD $(\geq 2$ vessels) were both higher in FMD $<6 \%$ groups, compared with $\mathrm{FMD} \geq 6 \%$ groups. Among the four groups, the highest incidence of multi-vessel CAD was seen in the FMD $<6 \% / \mathrm{RHI}<1.67$ group $(52 \%$ of patients in this group) (Table 1 ).

\section{Prediction of multi-vessel CAD using FMD and RHI}

In all 131 patients, multi-vessel CAD was seen in $34(26.0 \%)$ patients. Logistic regression analysis was performed to try to discriminate the 


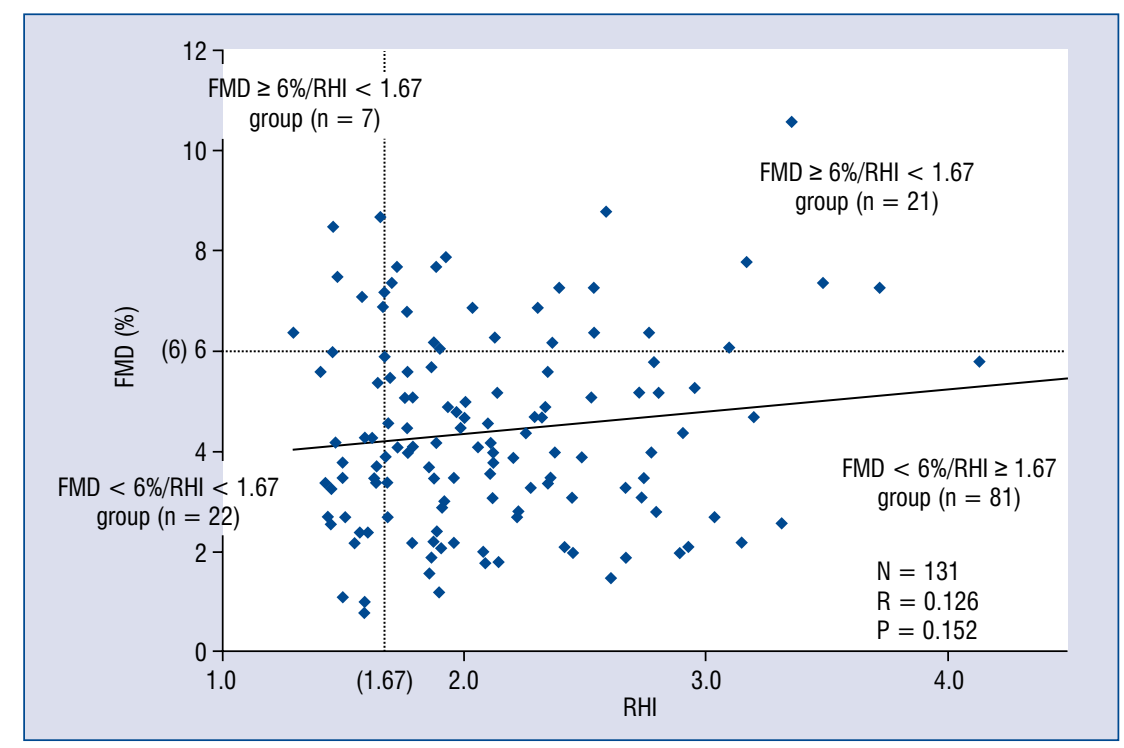

Figure 1. The relationship between flow-mediated dilation (FMD) and reactive hyperemia index (RHI) measured by reactive hyperemia-peripheral arterial tonometry in all patients. There was no significant correlation between FMD and RHI. Then, patients were divided into four groups, according to cut-off values of $6 \%$ for FMD and 1.67 for RHI: FMD $\geq 6 \%$ / $/ \mathrm{RHI} \geq 1.67$ group, $\mathrm{FMD} \geq 6 \% / \mathrm{RHI}<1.67$ group, $\mathrm{FMD}<6 \% / \mathrm{RHI} \geq 1.67$ group and $\mathrm{FMD}<6 \% / \mathrm{RHI}<1.67$ group.

34 multi-vessel CAD patients from the remaining 97 patients who had no coronary artery stenosis or just single-vessel disease. In simple logistic regression analysis, age, gender, coronary risk factors and vascular endothelial function parameters to predict multi-vessel disease were used. These analyses showed that the prevalence of both FMD $<6 \%$ and $\mathrm{RHI}<1.67$ was a significant predictor of multivessel disease (odds ratio [OR]: 4.161, 95\% confidence interval [CI]: 1.574-10.998, $\mathrm{p}=0.004)$; and age (OR: $1.026,95 \%$ CI: 0.986-1.067, $\mathrm{p}=0.206$ ), BMI (OR: 1.077, 95\% CI: 0.974-1.190, $\mathrm{p}=0.149$ ), systolic blood pressure (OR: 1.020, 95\% CI: 0.996$-1.045, \mathrm{p}=0.109$ ), hemoglobin A1c (OR: 1.439, 95\% CI: $0.892-2.322, \mathrm{p}=0.136)$ and eGFR (OR: $0.981,95 \%$ CI: $0.957-1.006, p=0.128)$ were potential predictors $(\mathrm{p}<0.3$ ) (Fig. 2). Multiple logistic regression analysis using all of these variables showed that only the prevalence of both FMD $<6 \%$ and $\mathrm{RHI}<1.67$ independently predicted multivessel CAD (OR: 4.160, 95\% CI: 1.505-11.500, $\mathrm{p}=0.006)($ Table 2$)$.

\section{RHI and the other parameters measured with FMD}

Next, other parameters measured during the FMD procedure separately in 29 patients with $\mathrm{FMD} \geq 6 \%$ and 102 patients with $\mathrm{FMD}<6 \%$ were assessed. In the patients with $\mathrm{FMD} \geq 6 \%$, there were no significant differences in baseline vessel diameter, maximum vessel diameter and blood flow velocity increase between 22 patients with $\mathrm{RHI}$ $\geq 1.67$ and 7 patients with $\mathrm{RHI}<1.67$. In patients with $\mathrm{FMD}<6 \%$, there were also no significant differences in these other parameters among 81 patients with $\mathrm{RHI} \geq 1.67$ and 21 patients with RHI < 1.67 (Table 3). However, RHI was negatively correlated with the baseline vessel diameter $(\mathrm{R}=-0.268, \mathrm{p}=0.0065)$ and maximum vessel diameter $(\mathrm{R}=-0.266, \mathrm{p}=0.0069)$ in the patients with $\mathrm{FMD}<6 \%$, whereas these correlations were absent in patients with FMD $\geq 6 \%$ (Fig. 3).

\section{Discussion}

In the present study, it was first demonstrated that FMD and RHI were not correlated in patients who underwent coronary angiography for the suspicion of CAD. Next, patients were divided into four groups, based on cut off values of $6 \%$ for FMD and 1.67 for RHI. As a result, $62 \%$ of the patients showed FMD $<6 \%$ but RHI $\geq 1.67$, and $52 \%$ of patients with FMD $<6 \%$ and $\mathrm{RHI}<1.67$ had multi-vessel CAD. Multiple regression analysis showed that the prevalence of both FMD $<6 \%$ and RHI $<1.67$ independently predicted multi-vessel CAD. Finally, the relationship was assessed between other parameters measured during the FMD procedure and RHI. As a result, $\mathrm{RHI}$ was negatively correlated with brachial artery diameter in patients 
Table 1. Baseline characteristics.

\begin{tabular}{|c|c|c|c|c|c|}
\hline & $\begin{array}{c}\text { Normal FMD/ } \\
\text { /normal RHI } \\
\text { (n= 22) }\end{array}$ & $\begin{array}{c}\text { Normal FMD/ } \\
\text { /low RHI } \\
(\mathbf{n}=7)\end{array}$ & $\begin{array}{l}\text { Low FMD/ } \\
\text { /normal RHI } \\
\text { (n=81) }\end{array}$ & $\begin{array}{l}\text { Low FMD/ } \\
\text { /low RHI } \\
\text { (n= 21) }\end{array}$ & $\mathbf{P}$ \\
\hline Age [years] & $64 \pm 10$ & $70 \pm 7$ & $67 \pm 12$ & $69 \pm 8$ & 0.413 \\
\hline Male gender & $17(77 \%)$ & $5(71 \%)$ & $62(77 \%)$ & $20(95 \%)$ & 0.266 \\
\hline Body mass index $\left[\mathrm{kg} / \mathrm{m}^{2}\right]$ & $25.2 \pm 4.1$ & $25.5 \pm 3.3$ & $23.6 \pm 3.6$ & $24.8 \pm 3.2$ & 0.137 \\
\hline \multicolumn{6}{|l|}{ Risk factors: } \\
\hline Hypertension & $16(73 \%)$ & $5(71 \%)$ & $55(68 \%)$ & $13(62 \%)$ & 0.891 \\
\hline Diabetes & $10(45 \%)$ & $2(29 \%)$ & $36(44 \%)$ & $14(67 \%)$ & 0.218 \\
\hline Dyslipidemia & $19(83 \%)$ & $19(83 \%)$ & $12(80 \%)$ & $12(80 \%)$ & 0.207 \\
\hline Smoking & $16(72 \%)$ & $4(57 \%)$ & $53(65 \%)$ & $14(67 \%)$ & 0.874 \\
\hline Systolic blood pressure [mmHg] & $131 \pm 17$ & $128 \pm 14$ & $134 \pm 18$ & $132 \pm 14$ & 0.959 \\
\hline LDL-cholesterol [mg/dL] & $94 \pm 23$ & $79 \pm 13$ & $82 \pm 23$ & $78 \pm 24$ & 0.103 \\
\hline HDL-cholesterol [mg/dL] & $48 \pm 13$ & $43 \pm 12$ & $50 \pm 14$ & $45 \pm 13$ & 0.911 \\
\hline Triglyceride [mg/dL] & $111 \pm 75$ & $74 \pm 38$ & $88 \pm 52$ & $75 \pm 57$ & 0.166 \\
\hline Hemoglobin A1c [\%] & $6.3 \pm 0.9$ & $6.1 \pm 0.5$ & $6.3 \pm 0.8$ & $6.5 \pm 0.7$ & 0.700 \\
\hline Creatinine [mg/dL] & $0.79 \pm 0.16$ & $0.77 \pm 0.17$ & $0.8 \pm 0.2$ & $0.9 \pm 0.2$ & 0.226 \\
\hline eGFR $\left[\mathrm{mL} / \mathrm{min} / 1.73 \mathrm{~m}^{2}\right]$ & $75.4 \pm 15.1$ & $71.8 \pm 9.3$ & $73.1 \pm 17.2$ & $67.2 \pm 16.6$ & 0.399 \\
\hline \multicolumn{6}{|l|}{ Medications: } \\
\hline ACEI/ARBs & $8(36 \%)$ & $5(71 \%)$ & $33(41 \%)$ & $7(33 \%)$ & 0.090 \\
\hline Sulfonylureas & $3(14 \%)$ & $0(0 \%)$ & $10(12 \%)$ & $3(14 \%)$ & 0.777 \\
\hline Insulins & $1(5 \%)$ & $0(0 \%)$ & $3(4 \%)$ & $0(0 \%)$ & 0.762 \\
\hline Statins & $16(73 \%)$ & $6(86 \%)$ & $64(79 \%)$ & $21(100 \%)$ & 0.096 \\
\hline \multicolumn{6}{|l|}{ Basal disease: } \\
\hline Stable angina pectoris & $6(27 \%)$ & $6(86 \%)$ & $21(68 \%)$ & $5(23 \%)$ & 0.009 \\
\hline Old myocardial infarction & $11(50 \%)$ & $1(14 \%)$ & $48(59 \%)$ & $16(76 \%)$ & 0.030 \\
\hline Coronary spastic angina & $4(18 \%)$ & $0(0 \%)$ & 7 (9\%) & $0(0 \%)$ & 0.150 \\
\hline Chest pain syndrome & $1(5 \%)$ & $0(0 \%)$ & $5(6 \%)$ & $0(0 \%)$ & 0.611 \\
\hline \multicolumn{6}{|l|}{ Affected vessel number: } \\
\hline No stenotic lesion & $5(23 \%)$ & $0(0 \%)$ & $12(15 \%)$ & $0(0 \%)$ & 0.099 \\
\hline Single vessel disease & $16(73 \%)$ & $4(57 \%)$ & $49(60 \%)$ & $10(48 \%)$ & 0.364 \\
\hline Multi-vessel disease & $1(5 \%)$ & $3(43 \%)$ & $20(25 \%)$ & $11(52 \%)$ & 0.005 \\
\hline
\end{tabular}

ACEI — angiotensin converting enzyme inhibitors; ARB — angiotensin receptor blocker; eGFR — estimated glomerular filtration rate; FMD — flow-mediated dilation; HDL — high density lipoprotein; LDL — low density lipoprotein; RHI — reactive hyperemia index

with low FMD, whereas a correlation was absent in patients with normal FMD.

Flow-mediated dilation and RH-PAT are both extremely effective noninvasive methods of evaluating vascular endothelial function, but have some physiological and clinical differences that depend on the vessels evaluated with each method. There are only a few studies that examined the relationship between FMD and RH-PAT based on RHI. Dhindsa et al [18]. demonstrated that FMD was significantly and positively associated with RHI in apparently healthy normotensive subjects. The Framingham Heart Study demonstrated that
FMD and RHI were not correlated in multivariable models [19], whereas the Gutenberg Heart Study showed a modest correlation [20]. However, in both of these studies, FMD, which represents endothelial function of large conduit arteries, and was particularly sensitive to impairment by traditional risk factors. In contrast, RHI, which represents endothelial function of resistance vessels, was more sensitive to metabolic risk factors, such as diabetes and obesity. Moreover, endothelial dysfunction in the conduit arteries and resistance vessels could also reflect different stages of atherosclerosis. Endothelial dysfunction in conduit arteries might 


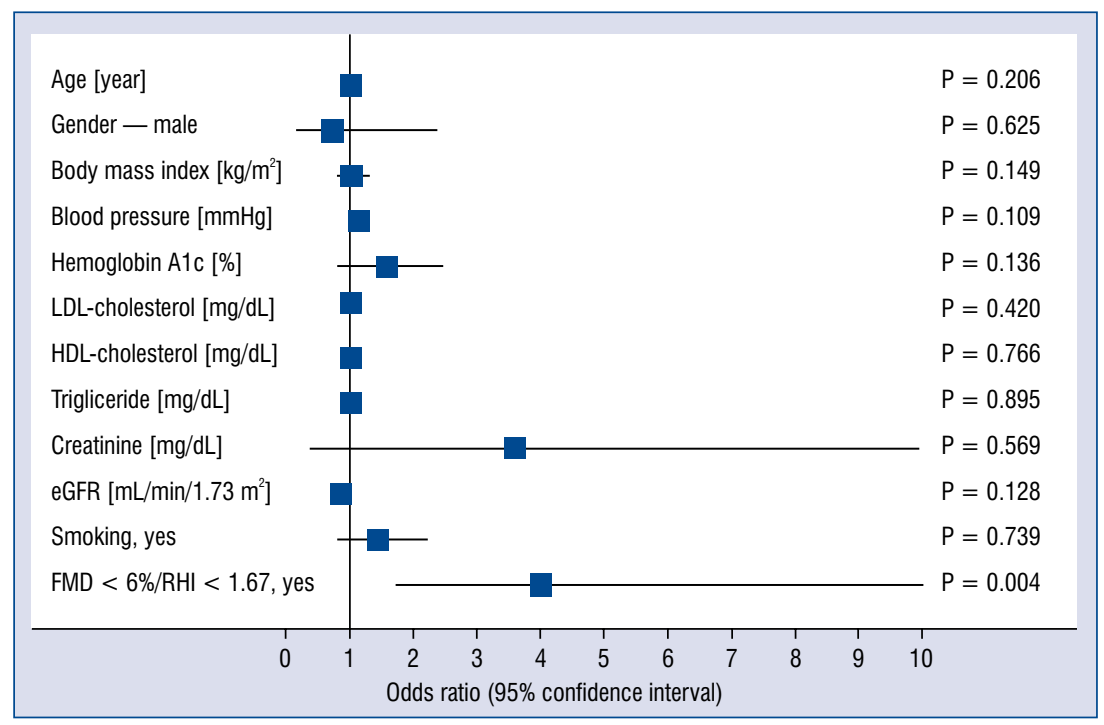

Figure 2. Simple logistic regression model to determine variables that predicted multi-vessel coronary artery disease. The prevalence of both flow-mediated dilation (FMD) $<6 \%$ and reactive hyperemia index (RHI) $<1.67$ was a significant predictor of multi-vessel disease; age, body mass index, systolic blood pressure, hemoglobin A1c and estimated glomerular filtration rate (eGFR) were also potential predictors of multi-vessel disease; HDL — high density lipoprotein; LDL - low density lipoprotein.

Table 2. Multiple logistic regression analysis for prediction of multi-vessel coronary artery disease.

\begin{tabular}{lcccc}
\hline & Wald $\lambda^{2}$ & Odds ratio & $95 \% \mathrm{Cl}$ & $\mathbf{P}$ \\
\hline Age $[$ years] & 0.609 & 1.002 & $0.968-1.079$ & 0.435 \\
Body mass index $\left[\mathrm{kg} / \mathrm{m}^{2}\right]$ & 1.151 & 1.068 & $0.947-1.204$ & 0.283 \\
Systolic blood pressure $[\mathrm{mmHg}]$ & 1.133 & 1.016 & $0.987-1.045$ & 0.287 \\
Hemoglobin A1c $[\%]$ & 1.200 & 1.346 & $0.791-2.289$ & 0.273 \\
eGFR $\left[\mathrm{mL} / \mathrm{min} / 1.73 \mathrm{~m}^{2}\right]$ & 0.183 & 0.993 & $0.964-1.024$ & 0.669 \\
FMD $<6 \% / \mathrm{RHI}<1.67$, yes & 7.549 & 4.160 & $1.505-11.500$ & 0.006 \\
\hline
\end{tabular}

$\mathrm{Cl}$ — confidence interval; eGFR — estimated glomerular filtration rate; FMD — flow-mediated dilation; RHI — reactive hyperemia index

Table 3. Other parameters measured during flow-mediated dilation (FMD) procedure.

\begin{tabular}{|c|c|c|c|c|c|c|}
\hline & \multicolumn{3}{|c|}{ FMD $\geq 6 \%(n=29)$} & \multicolumn{3}{|c|}{ FMD $<6 \%(n=102)$} \\
\hline & $\begin{array}{c}\mathrm{RHI} \geq 1.67 \\
(\mathrm{n}=22)\end{array}$ & $\begin{array}{c}\mathrm{RHI}<1.67 \\
(\mathrm{n}=7)\end{array}$ & $\mathbf{P}$ & $\begin{array}{c}\mathrm{RHI} \geq 1.67 \\
(\mathrm{n}=81)\end{array}$ & $\begin{array}{c}\mathrm{RHI}<1.67 \\
(\mathrm{n}=21)\end{array}$ & $\mathbf{P}$ \\
\hline Baseline vessel diameter [mm] & $3.93 \pm 0.53$ & $3.69 \pm 0.60$ & 0.313 & $4.31 \pm 0.56$ & $4.46 \pm 0.64$ & 0.313 \\
\hline Maximum vessel diameter $[\mathrm{mm}]$ & $4.21 \pm 0.56$ & $3.95 \pm 0.63$ & 0.314 & $4.47 \pm 0.57$ & $4.60 \pm 0.64$ & 0.380 \\
\hline Blood flow velocity increase & $5.68 \pm 2.42$ & $4.17 \pm 1.46$ & 0.133 & $4.83 \pm 2.00$ & $4.93 \pm 2.55$ & 0.844 \\
\hline
\end{tabular}

$\mathrm{RHI}$ - reactive hyperemia index

be more important in patients with existing atherosclerosis, whereas that in resistance vessels might be an early indicator of arteriosclerosis risk [19-21]. Recently, Tomiyama et al. [14] found no correlation between FMD and RH-PAT when the two parameters were measured simultaneously, and results were similar to the present results. They demonstrated that autonomic nervous activation, especially sympathetic nerve activation induced by reactive hyperemia, affected RHI more than FMD. 

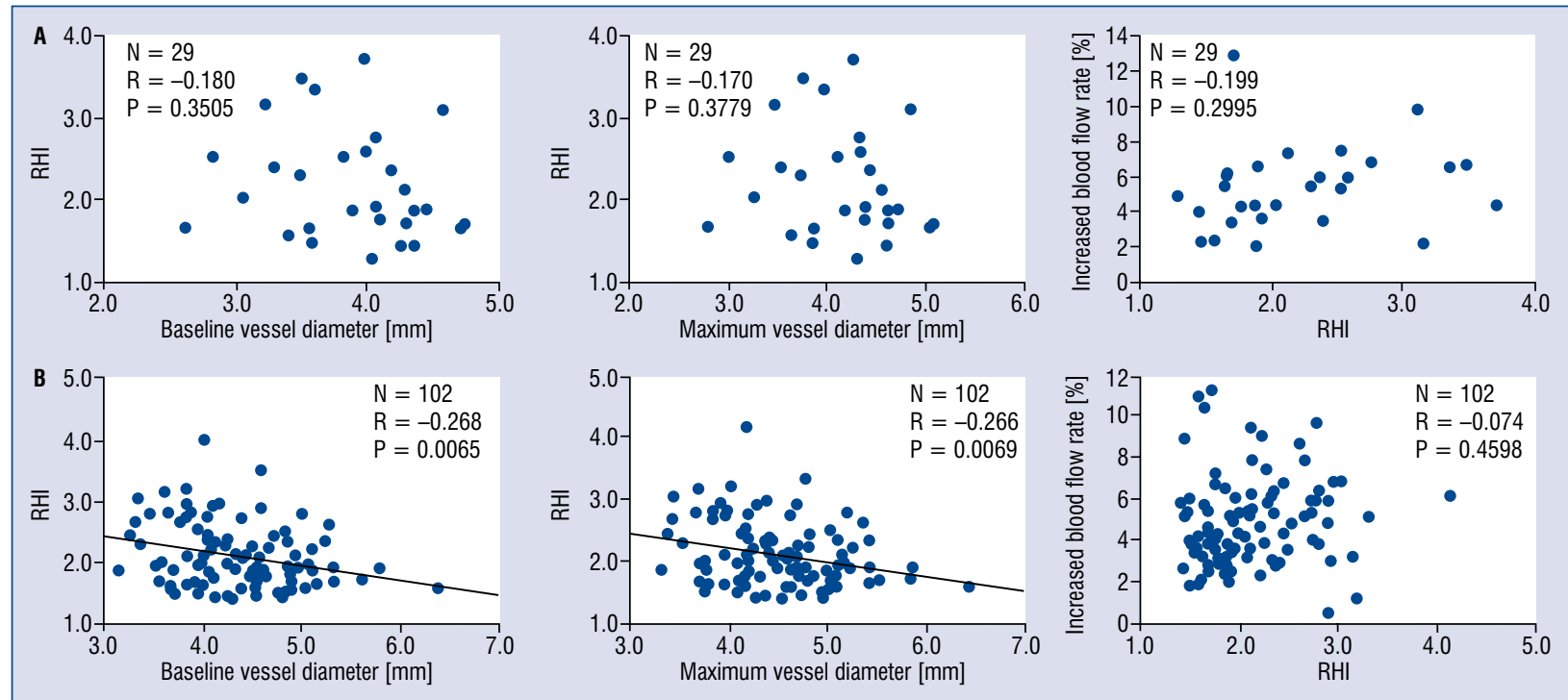

Figure 3. The correlation between reactive hyperemia index (RHI) and the other parameters obtained during flow-mediated dilation (FMD) procedure. A. In patients with $\mathrm{FMD} \geq 6 \%$, there were no significant correlations; $\mathbf{B}$. In patients with $\mathrm{FMD}<6 \%$, however, $\mathrm{RHI}$ was negatively correlated with the baseline vessel diameter and maximum vessel diameter.

Although the relationship between FMD and $\mathrm{RHI}$ is controversial as described above, there was no correlation between these two parameters In all patients in the present study. However, analysis herein, after dividing patients into four groups provided some new information. Patients with stenotic lesions (i.e., coronary spastic angina or chest pain syndrome) were present in groups with low RHI. In addition, the highest incidence of multi-vessel CAD was seen in the group with the prevalence of both FMD $<6 \%$ and RHI $<1.67$. This result suggests that the presence of low values of both FMD and RHI would be a strong risk factor for severe CAD. Furthermore, multiple regression analysis showed that the incidence of both FMD $<6 \%$ and RHI $<1.67$ could independently predict multi-vessel $\mathrm{CAD}$ also supports this suggestion. Woo et al. [22] compared FMD and RHI in patients with $\mathrm{CAD}$ and demonstrated that the value of each parameter was significantly lower in patients with multi-vessel and complex CAD. Their receiver-operating characteristic curve analysis showed that both FMD and RHI had a similar value for predicting the presence of $\mathrm{CAD}$ and its complexity. They concluded that RHPAT would be a more useful method for evaluating vascular endothelial function in patients with $\mathrm{CAD}$, because it is less operator-dependent and it is noninvasive compared with FMD. According to the present results, however, it is envisioned herein that the assessment of vascular endothelial functions of both conduit arteries and resistance vessels using the simultaneous measurement of FMD and RH-PAT would be more sensitive for the prediction of severe CAD.

Another novel finding of the present study is that RHI was negatively correlated with brachial artery diameter at baseline as well as at the maximum response after forearm cuff deflation following compression in patients with $\mathrm{FMD}<6 \%$, whereas these correlations were absent in patients with FMD $\geq 6 \%$. Thus, lower RHI is associated with larger baseline brachial artery diameter, since the change in vessel diameter at maximum response after forearm cuff deflation was small in cases with low FMD. This result suggests that reactive hyperemic flow response of resistance vessels might depend on vascular remodeling in the conduit artery. Endothelial function contributes to the maintenance of vasodilator tone by endotheliumdependent relaxing factors, including $\mathrm{NO}$ and $\mathrm{EDHF}$ $[11,12]$. Endothelium-dependent vasodilation in the conduit artery is mediated mainly by NO, whereas the dilation of resistance vessels is mediated by $\mathrm{NO}$ and EDHF together [23]. In patients with low FMD, basal NO bioavailability might decrease. Since decreased NO bioavailability leads to positive vessel remodeling [24], it is postulated that EDHF contributes more to the endothelium-dependent vascular response in resistance vessels than $\mathrm{NO}$ in $\mathrm{CAD}$ patients with low FMD. 


\section{Limitations of the study}

The present study has several potential limitations. First, the sample size was too small to draw definitive conclusions. Although it was suggested that both low FMD and low RHI are risk factors for severe $\mathrm{CAD}$, the number of patients in this group was small. In addition, a negative correlation was observed between RHI and brachial artery diameter in patients with FMD $<6 \%$, but not in those with $\mathrm{FMD} \geq 6 \%$. However, the number of patients with $\mathrm{FMD} \geq 6 \%$ was small $(\mathrm{n}=29)$, whereas there were 81 patients with FMD $<6 \%$, so the absence of a correlation in patients with FMD $\geq 6 \%$ may be a type II error. Further assessment using a larger number of patients is needed. Second, this study was a cross-sectional study, and logistic regression analysis was used to discriminate patients with multi-vessel disease from others. To evaluate the value of FMD and RH-PAT to predict severe CAD, a prospective observational study is required. Finally, the mechanism for correlation between RHI and brachial artery diameter in patients with FMD $<6 \%$ may be somewhat speculative. Even considering these limitations, however, it is believed that this study showed important and clinically significant differences between FMD and RHI, and the advantages of simultaneous measurement of both values.

\section{Conclusions}

From the present study it can be envisioned that noninvasive assessment of vascular endothelial functions provide pathophysiological information on both conduit arteries and resistance vessels, and that this information is relevant to CAD. A unique classification by simultaneous measurement of FMD and RHI would allow the prediction of the severity of the coronary artery lesions and the presence of advanced atherosclerotic CAD.

\section{Conflict of interest: None declared}

\section{References}

1. Lerman A, Zeiher AM. Endothelial function: cardiac events. Circulation. 2005; 111(3): 363-368, doi: 10.1161/01.CIR.0000153339. 27064.14, indexed in Pubmed: 15668353.

2. Flammer AJ, Anderson T, Celermajer DS, et al. The assessment of endothelial function: from research into clinical practice. Circulation. 2012; 126(6): 753-767, doi: 10.1161/CIRCULATIONAHA.112.093245, indexed in Pubmed: 22869857.

3. Anderson TJ, Uehata A, Gerhard MD, et al. Close relation of endothelial function in the human coronary and peripheral circulations. J Am Coll Cardiol. 1995; 26(5): 1235-1241, indexed in Pubmed: 7594037.
4. Celermajer DS, Sorensen KE, Gooch VM, et al. Non-invasive detection of endothelial dysfunction in children and adults at risk of atherosclerosis. Lancet. 1992; 340(8828): 1111-1115, indexed in Pubmed: 1359209.

5. Joannides R, Haefeli WE, Linder L, et al. Nitric oxide is responsible for flow-dependent dilatation of human peripheral conduit arteries in vivo. Circulation. 1995; 91(5): 1314-1319, indexed in Pubmed: 7867167.

6. Anderson TJ, Uehata A, Gerhard MD, et al. Close relation of endothelial function in the human coronary and peripheral circulations. J Am Coll Cardiol. 1995; 26(5): 1235-1241, indexed in Pubmed: 7594037.

7. Green DJ, Jones H, Thijssen D, et al. Flow-mediated dilation and cardiovascular event prediction: does nitric oxide matter? Hypertension. 2011; 57(3): 363-369, doi: 10.1161/HYPERTENSIONAHA.110.167015, indexed in Pubmed: 21263128.

8. Matsubara J, Sugiyama S, Akiyama E, et al. Dipeptidyl peptidase-4 inhibitor, sitagliptin, improves endothelial dysfunction in association with its anti-inflammatory effects in patients with coronary artery disease and uncontrolled diabetes. Circ J. 2013; 77(5): 1337-1344, indexed in Pubmed: 23386232.

9. Inoue T, Matsuoka H, Higashi $\mathrm{Y}$, et al. Flow-mediated vasodilation as a diagnostic modality for vascular failure. Hypertens Res. 2008; 31(12): 2105-2113, doi: 10.1291/hypres.31.2105, indexed in Pubmed: 19139600.

10. Hamburg NM, Palmisano J, Larson MG, et al. Relation of brachial and digital measures of vascular function in the community: the Framingham heart study. Hypertension. 2011; 57(3): 390-396, doi: 10.1161/HYPERTENSIONAHA.110.160812, indexed in Pubmed: 21263120.

11. Nohria A, Gerhard-Herman M, Creager MA, et al. Role of nitric oxide in the regulation of digital pulse volume amplitude in humans. J Appl Physiol (1985). 2006; 101(2): 545-548, doi: 10.1152/ japplphysiol.01285.2005, indexed in Pubmed: 16614356.

12. Vanhoutte PM, Mombouli JV. Vascular endothelium: vasoactive mediators. Prog Cardiovasc Dis. 1996; 39(3): 229-238, indexed in Pubmed: 8970575.

13. Rubinshtein R, Kuvin JT, Soffler M, et al. Assessment of endothelial function by non-invasive peripheral arterial tonometry predicts late cardiovascular adverse events. Eur Heart J. 2010; 31(9): 1142-1148, doi: 10.1093/eurheartj/ehq010, indexed in Pubmed: 20181680.

14. Tomiyama H, Yoshida M, Higashi Y, et al. Autonomic nervous activation triggered during induction of reactive hyperemia exerts a greater influence on the measured reactive hyperemia index by peripheral arterial tonometry than on flow-mediated vasodilatation of the brachial artery in patients with hypertension. Hypertens Res. 2014; 37(10): 914-918, doi: 10.1038/hr.2014.103, indexed in Pubmed: 24942768.

15. Matsuo S, Imai E, Horio M, et al. Collaborators developing the Japanese equation for estimated GFR. Revised equations for estimated GFR from serum creatinine in Japan. Am J Kidney Dis. 2009; 53(6): 982-992, doi: 10.1053/j.ajkd.2008.12.034, indexed in Pubmed: 19339088.

16. Vogel RA. Measurement of endothelial function by brachial artery flow-mediated vasodilation. Am J Cardiol. 2001; 88(2A): 31E-34E, indexed in Pubmed: 11473743.

17. Bonetti PO, Pumper GM, Higano ST, et al. Noninvasive identification of patients with early coronary atherosclerosis by assessment of digital reactive hyperemia. J Am Coll Cardiol. 2004; 44(11): 2137-2141, doi: 10.1016/j.jacc.2004.08.062, indexed in Pubmed: 15582310. 
18. Dhindsa M, Sommerlad SM, DeVan AE, et al. Interrelationships among noninvasive measures of postischemic macro- and microvascular reactivity. J Appl Physiol (1985). 2008; 105(2): 427-432, doi: 10.1152/japplphysiol.90431.2008, indexed in Pubmed: 18483158

19. Hamburg NM, Palmisano J, Larson MG, et al. Relation of brachial and digital measures of vascular function in the community: the Framingham heart study. Hypertension. 2011; 57(3): 390-396, doi: 10.1161/HYPERTENSIONAHA.110.160812, indexed in Pubmed: 21263120.

20. Schnabel RB, Schulz A, Wild PS, et al. Noninvasive vascular function measurement in the community: cross-sectional relations and comparison of methods. Circ Cardiovasc Imaging. 2011; 4(4): 371-380, doi: 10.1161/CIRCIMAGING.110.961557, indexed in Pubmed: 21551420.

21. Hamburg NM, Keyes MJ, Larson MG, et al. Cross-sectional relations of digital vascular function to cardiovascular risk fac- tors in the Framingham Heart Study. Circulation. 2008; 117(19): 2467-2474, doi: 10.1161/CIRCULATIONAHA.107.748574, indexed in Pubmed: 18458169.

22. Woo JS, Jang WS, Kim HS, et al. Comparison of peripheral arterial tonometry and flow-mediated vasodilation for assessment of the severity and complexity of coronary artery disease. Coron Artery Dis. 2014; 25(5): 421-426, doi: 10.1097/ MCA.0000000000000094, indexed in Pubmed: 24584030.

23. Schiffrin EL. Beyond blood pressure: the endothelium and atherosclerosis progression. Am J Hypertens. 2002; 15(10 Pt 2): 115S-122S, indexed in Pubmed: 12383592.

24. Tong XK, Nicolakakis N, Kocharyan A, et al. Vascular remodeling versus amyloid beta-induced oxidative stress in the cerebrovascular dysfunctions associated with Alzheimer's disease. J Neurosci. 2005; 25(48): 11165-11174, doi: 10.1523/JNEUROSCI.4031-05.2005, indexed in Pubmed: 16319316. 